\title{
A simulation model for building use re-thinking after the COVID-19 emergency
}

XXIV International Conference of the Iberoamerican Society of Digital Graphics Medellin | Colombia

\author{
Davide Simeone \\ Sapienza University of Rome | Italy | davide.simeone@uniroma1.it \\ Antonio Fioravanti \\ Sapienza University of Rome | Italy | antonio.fioravanti@uniroma1.it \\ Ugo Maria Coraglia \\ Sapienza University of Rome | Italy | ugomaria.coraglia@uniroma1.it \\ Stefano Cursi \\ Institute of Heritage Science | Italy | stefano.cursi@ispc.cnr.it
}

\begin{abstract}
COVID-19 infection is forcing designers and building managers in re-thinking the use and experience of architectural spaces, as well as the interactions within the people in it. To support this difficult task, this research is working on a simulation model, based on agent-based modeling, able to predict the use phenomena of buildings and provided quantitative and qualitative feedback regarding the impact of re-defined use processes to COVID-19 infection risk. The derived platform is particularly designed to support the testing of visiting scenarios in museums and galleries, potentially helping them in their re-opening phases.
\end{abstract}

Keywords: Agent-based modeling and simulation (ABMS); COVID-19; Building use; Behavioral simulation; Unity 3D.

\section{RATIONALE \\ COVID-19 CONTEXT AND IMPACT ON BEHAVIOR IN BUILDINGS}

The recent emergency of COVID-19 spreading virus has deeply impacted the way we were used to conceiving and shaping the behavior of people in buildings. After the initial lockdown period in the acute phase of the virus pandemic, where the use of the majority of buildings and public spaces has been close to zero, the gradual re-gaining of the use of our buildings is facing a deep transformation in terms of use and experiences, leaving aside consolidated design and spaces organization paradigms in favor of new ways, partially unexplored, to ensure both risk-free spaces and productive activities. For instance, a recent McKinsey report (2020) depicts an unprecedented situation for offices, with more people working from home and companies required to redesign spaces to ensure physical distancing, avoid congestions in the different areas and guarantee high levels of air quality and recirculation. This is just part of a way much larger framework that includes strong shifts in terms of companies' culture, work processes, productivity philosophy, collaboration attitude and so forth, but the redesign of the way our buildings will be used and experienced is probably the major task that the architectural design sector will face in the next few months. Same difficulties are being experienced in other domains of the built environment: World Health Organization has published policies and guidelines to support schools re-opening (2020), while other public institutions (in particular museums) are experiencing difficulties in trying to fulfill national requirements and scientific prescriptions with a $13 \%$ risk of permanent closures (UNESCO report, 2020; ICOM report, 2020). An important debate is also dealing with the impact on public spaces, although less at risk of virus spreading (HoneyRoses, 2020).

This process of rethinking and redesign spaces and their use is facing two main challenges: on one side, there is the absence of historic data that can support the design decisions, allowing for an assessment of design in terms of infection risks, although some protocols have emerged there is still much uncertainty on which extend take into account general assumptions in terms of social distancing. On the other side, this rethinking process is required to happen in a short amount of time, probably some months, to ensure as soon as possible productivity of offices, the profitability of real estate and accessibility of public spaces and cultural institutions.

\section{SOCIAL DISTANCING AND OTHER SPACES USE ASSUMPTIONS FOR COVID RISK REDUCTION}

Since the beginning of the COVID-19 epidemic, several key aspects have progressively emerged as influencing the spreading of the virus both in closed and open spaces: physical contacts, proximity and exposure time in social interactions, use of masks and adoption of personal protective equipment, spending a large part of the day in an environment with a positive case (for instance at home), and having direct contact with healthcare personnel dedicated with the direct or indirect treatment of COVID-19 (Report COVID-19 - Che cos'è il nuovo coronavirus, 2020). Among these, three are the factors that are particularly significant in terms of rethinking the use of spaces, and that we considered relevant for this research:

1. Prolonged exposure ( $>15$ minutes) at less than 2 meters distance.

2. Prolonged co-presence in closed spaces, and

3. Direct physical contact. 
Nevertheless, the values of these parameters are continuously being updated by research, norms and regulations and vary depending on the social, cultural and geographical context considered.

\section{USERS' BEHAVIOR SIMULATION AS A WAY OF PREDICTING COVID-19 RISK}

In this unprecedented attempt of re-thinking the intended use processes and modalities of our buildings, a crucial role can be played by the use of simulative approaches to digitally predict and assess new use scenarios, supporting decisions of both designers and owners and allowing them to rapidly test new protocols and environments modifications before actually stepping into the building or starting renovation works. In fact, by virtually simulating the behavior of users in the building, and by measuring key parameters such as the time that each agent is at a risky distance from other agents, it is possible to compare different solutions both in terms of changes in the activities to be hosted and to the environment itself. This comparison allows the decision-makers to be more aware of the actual impact of their choices and to balance risks and productivity both in short and long terms (Simeone, 2012).

For this purpose, this research presents a use simulation model, based on agent-based modeling and oriented to simulation of use patterns in public buildings, and that allows for rapid testing of redefined use processes to provide specific feedbacks related to potential risks for COVID-19 spreading. Considered the complexity of Virus spreading phenomena as well as its uncertainties, the proposed model relies on the essential parameter of mutual people distancing and exposure time to verify and measure how many interactions or proximity under this range occurs during the use of the building. As shown later in this paper, we chose to test the proposed simulation model in the context of cultural buildings such as museums, galleries, temporary exhibitions, to provide this suffering sector of a supportive approach and a dedicated tool to make the necessary decisions for a quick restart.

\section{STATE OF THE ART}

\section{AGENT-BASED AND BEHAVIORAL MODELING FOR OCCUPANCY SIMULATION}

In the CAAD field, much research has targeted the use of digital simulation to predict the complex phenomena of human behavior in buildings, with the clear objective of virtually assess design before stepping into the construction phase. In particular, the conception of the Agent-Based Modeling and Simulation (ABMS) paradigm (Axelrod, 1997; Bonabeau 2002) has led to a new set of applications for specific disciplines such as crowd modeling, evacuation and egress simulation (Chu, 2014), and pedestrian movement prediction, both indoor and in public spaces (Batty, 2001). While the ABMS approach has demonstrated its reliability in modeling use phenomena generated by the interaction of single, autonomous behaviors of agents, some limits have progressively emerged in accurately representing the complex systems of collective actions and activities that are the key elements of a building use process. For this reason, much research has integrated ABMS with activity-based modeling (Tabak, 2008; Wurzer, 2010), Event-based modeling (Simeone 2012) to have single behaviors driven and coordinated from a higher-level process perspective. In the specific area of cultural buildings, the different experiments have usually preferred pure ABMS systems to control and assess only people movements to mainly assess the capacity of the exhibition spaces (Pluchino, 2014) or other agentperspective phenomena such as visitors' visibility (Cancellar, 2017). From a tool perspective, there is a variety of platforms that have been used to develop these applications: game-engines as Unity 3D and Virtools, ABMS platform such as Netlogo, multimethod simulation modeling tool such as Anylogic and so forth.

\section{RECENT APPROACHES TO SIMULATE POST-COVID OCCUPANCY}

Before COVID-19 emergency, ABMS systems have already been used to study and assess epidemic spreading more at territorial and urban (Perez, 2009) scale, with less attention on spreading at the level of building/public spaces (i.e. in 2015 Goscé et al. presented an analytical model to predict infections spreading in confined, crowded spaces). The particular nature of the COVID-19 infection and the deep correlation with aspects such as social distancing and spaces crowding have pushed some research in looking into this other dimension of epidemics: D'Orazio (2020) applied ABMS to discuss the impact of different operational decisions on the risks of COVID infection in public buildings (such as the adoption of social distancing and masks); Ronchi (2020) proposed the EXPOSED model to include COVID-19 parameters in crowd simulation to compare different solutions in crowd management. At the same time, some software houses specialized in ABMS for pedestrian movement simulations in indoor and outdoor spaces, have introduced additional features to their already well-known products (i.e. Massmotion by Oasys-Arup or Smart Move by Buro Happold) to include social distancing in the simulation.

While the mentioned research is focusing on the use of $\mathrm{ABM}$ to check general strategies or to manage crowds, at present there is no evidence of research aimed at developing a more comprehensive approach and derived tools to control more complex use phenomena - different from the pedestrian movement within the building - and to support the difficult design balancing between modifications to the building and its spaces and the intended use and users of the building.

\section{THE SIMULATION APPROACH TO USE RETHINKING}

\section{METHODOLOGY}

The presented research investigated a different approach to digitally support the design and organizational decisions in relation with COVID-19 spreading in built environments - in particular within the scope of cultural buildings such as museums or art galleries - based on the adoption of behavioral simulation within a game engine environment. The game engine (in our case Unity 3D) acts as a middleware that integrates:

1. The model of the built environment, enriched with usability information and with all the elements (fixed furniture, etc.) that have an impact on the use processes.

2. The agents and their behavioral algorithms intended to represent the users and their movements/actions within the building.

3. A configurational editor to place elements that drive the agents' behavior (i.e. art pieces that attract visitors in a museum).

4. A COVID-19 assessment engine made of a set of customizable algorithms and parameters that 
control and measure the risk related to reduced proximity or co-presence in the same spaces.

The adoption of a game engine, used as a central platform for the integration of these different aspects, provided us with some advantages such as the good interoperability with current BIM authoring tools, the availability of a large set of $\mathrm{Al}$ resources (such as navigation and pathfinding intelligence for agents), the programming and customization capabilities that allow developing all the necessary algorithms within the game engine framework, and the possibility to implement user interfaces to allow also non-experts in programming to autonomously use the platform and perform their assessments. Our objective was to create a user-friendly platform for non-expert users to upload a simplified design model, set up the initial parameters and make easy space layout changes.

After the initial conception of the system and the realization of the prototype, we chose to adopt the proposed platform in some case studies related to the reconfiguration of cultural spaces in some relevant museums in Italy. The models of the buildings and the configuration of the art pieces have been reconstructed by the documentation usually available in the museums' website regarding the spatial layout and the visiting paths, while for agents' behavior rules we relied on the navigation component proposed by Unity 3D enriched with custom rules to adhere to the specificness of the visiting behavior. Regarding the COVID-19 risks parameter, we created a set of rules able to use parameters such as the social distance or the exposure time as a reference and to update them in accordance to changes in norms and regulations.

\section{COMPONENTS' KEY FEATURES AND INTEGRATION WITHIN THE GAME ENGINE}

As discussed in the methodology section, we focused on each of the parts that compose the proposed virtual simulation system, distributing Al resources (as well as attributes and parameters) to the elements of each area, to obtain more flexibility during the calibration phase and better computational efficiency.

The environment: To favor the integration of the proposed approach in the current design processes, we choose to adopt a workflow for importing the model of the built environment into the simulation platform rather than remodeling the building in the game engine. In particular, we choose to use a .fbx exporter from Autodesk Revit (fig.1 and 2), integrated with a dynamo algorithm for exporting parameters and attributes associated to the elements of the model while, on the Unity $3 \mathrm{D}$ side, we customized an already existing algorithm to re-associate the attributes to the entities in the game engine. The use of a dynamo algorithm also allows to filter and control the attributes typology to ensure manageability of the data exported and to be retrieved in the Unity $3 \mathrm{D}$ environment.

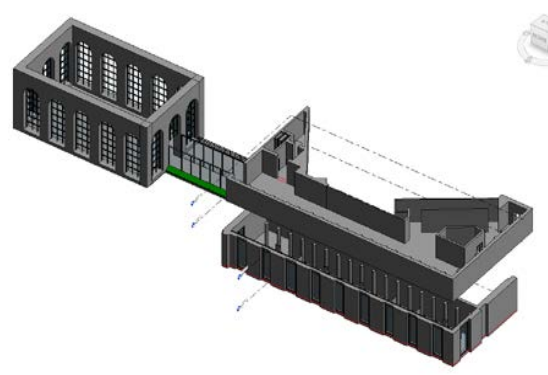

Figure 1: One of the case studies developed to test simulation accuracy in multi-story buildings (in this case the layout is derived by the visiting map of the "Museo del Novecento" in Milan).

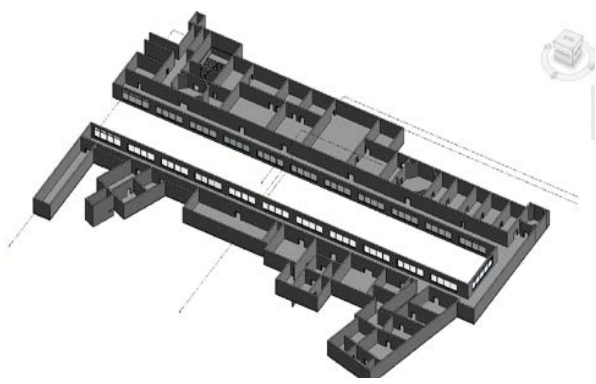

Figure 2: Another case study (developed relying on the layout of the Uffizi Gallery in Florence) used to simulate use scenarios and COVID-19 risk in sequences of rooms and confined spaces.

Although simplified, the building model is still composed of spaces and building components, each of them provided with their attributes and behavioral/measurement rules in order both to contribute in the simulation or to assess specific aspects of the simulated use (Simeone, 2019). For instance, space units can measure the number of people within their volume in each frame of the simulation, while doors can count the number of people passed through. When intervening on testing different scenarios, additional rules can control doors in not letting people's passing if the following space is too crowded, and so on. Essentially, while in real-world doors and other building components are mainly passive objects, in the virtual world can be provided with artificial intelligence to provide more accurate and reliable use scenarios (Simeone, 2017).

After the importing and the building model into the Unity 3D environment, a navigation map is created to identify all the reachable floor areas that users can walk on, while custom objects and moveable furniture do not impact the walkability map but are considered only in the avoidance management system of each agent, to optimize computational resources (fig.3). 


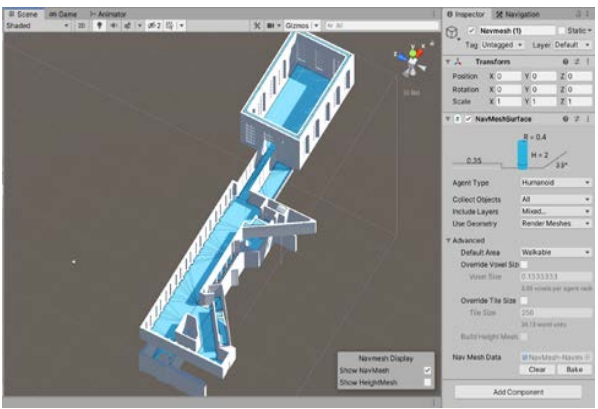

Figure 3: The navigation mesh generation (left) and the navigation setting (right) from one of the case studies.

Users as autonomous agents: As usual in the ABM application to the simulation of crowd simulation in the architecture field, users are represented as agents, autonomous systems with associated status and behavioral rules. To assess the human behavior for COVID-19 prevention, some modifications have been made to the status attributes and to the behavioral/navigation rules of the agents: in the agents' status area, an attribute regarding the exposure duration meant as the time spent at less than the safety distance from another visitor - is used to incrementally compute, through an algorithm, an indicator of the overall infection risk during the visiting.

Another important adjustment is made in the navigation and avoidance set of rules: avoidance of other agents is controlled by a customizable parameter and force the agent in avoiding performing navigation at less than the avoidance distance (i.e. 1 meter), and in finding new paths or stopping. Avoidance distance should be carefully reassessed before each simulation to increase the reliability of results, as per use choices (i.e. the use of masks). To increase accuracy, introducing the blurry of perception of the distance that usually occurs to any visitor, a distance noise parameter has been integrated into the algorithm, customizable depending on design and management choices (i.e. reduced in case of signals on the floor).

The use configurator: As part of the simulation inputs, a use configuration has to be provided by introducing in the model some elements that drive and influence the behavior of agents within the building. The elements are:

- Entrances and exits (where agents/users are generated and destroyed)

- Gates to drive agents' movements

In the context of museums and galleries, we chose to introduce the art piece entity meant as an attractor for the agents. In addition to the geometrical representation of the art piece (including if flat as paintings or tridimensional as sculptures), we added some parameters such as the optimal distance for its view and its degree of interest, that influence the crowding in its proximity.

To favor the usability from non-design experts as well as the flexibility during the experiment, we chose to design an easy interface that allows for direct placement and rotation of the art pieces as well as the control of the two parameters mentioned above.

This component allows the designer to test different solutions and scenarios, intervening both on the position of the art pieces and the number of users allowed at each time (fig. 4).

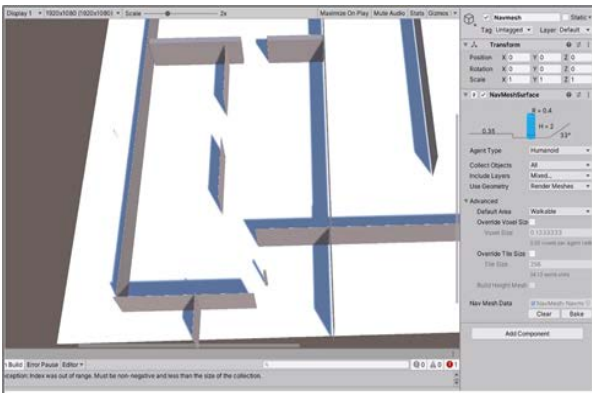

1. Building Model Import

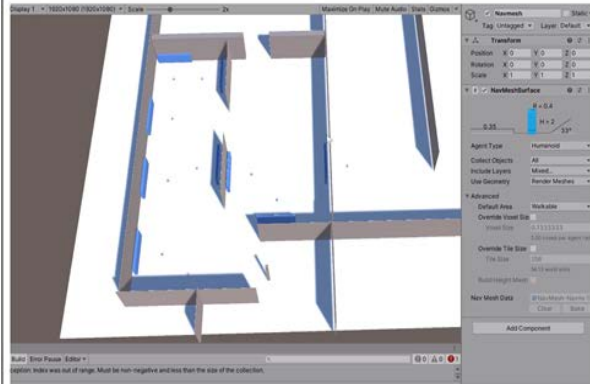

2. Configuration of use layout

Figure 4: Model Import (top) and use layout configuration (bottom) within the game engine environment.

The COVID-19 assessment engine: The overall estimation of the COVID-19 infection risk is delegated to the last component of the system - the assessment engine - that has the role of measuring the simulated use phenomena, performing some quantitative analysis and report them to allow for comparison of the effects of the design and management choices. In our case, the assessment engine collects data from the agents (such as the exposure time described before) or the duration of crowding in a specific space, interrelating such data with the input data (entrance frequency, imposed avoidance distance, etc.) providing a full understanding of the relationships with the use scenario and the infection risks (fig.5). 


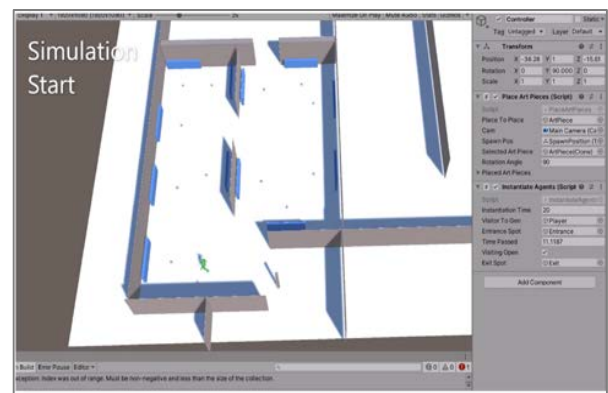

3. Simulation Start (Agents' instantiation)

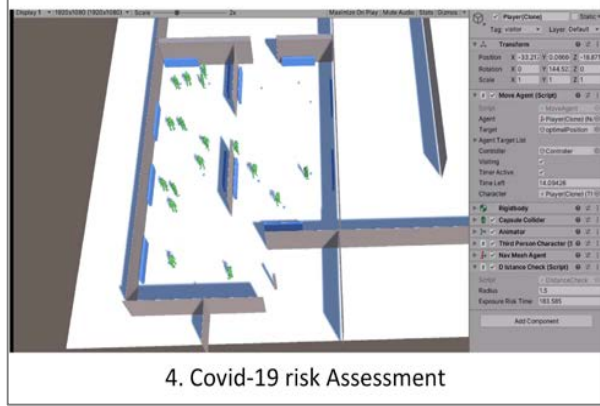

Figure 5: Performing of use simulation (top) and measurement of the COVID-19 risk parameters (bottom).

\section{THE TESTING OF THE PLATFORM FOR BUILDING OCCUPANCY RE-THINKING}

At present, the model is under testing for museums and other cultural buildings, to provide an easy and open platform that can support their progressive reopening. The platform allows to intervene on different aspects that influence the use processes, such as time gap between accesses, spaces layout, and distribution of the art pieces, and returning the seconds of risky proximity as per current social distancing rules. The platform is showing good flexibility in supporting use-related decisions and a better understanding of the real capabilities of exhibition spaces to host visitors in full respect of the regulations provided by norms and rules. The use of a middleware as Unity $3 D$ as the main simulation environment provides architectural designers to integrate this approach in the usual design processes, relying on its good interoperability with BIM modeling tools such as Autodesk Revit. Similarly, the configurator editor allows for a quick, intuitive re-design of the use process, allowing designers, owners, and curators to re-think the way the architectural space will be experienced by visitors, testing different configurations and intervening on different features of the intended scenarios and assessing the impact of each of them.

The use of a customizable parameter is another positive point in the adoption of the platform, that allows the designers and owners to assess the simulated use following reference values that are continuously changing depending on evolving research, context, governments' norms and regulations. The number of maximum people per square meter or the minimum distance between
Visitors are, for instance, parameters that are imposed by governments but still not standardized.

During the experiments we tested the integration with BIM at different Level of Development (LoD), assessing an overall equivalence in terms of accuracy of simulated results and a preference for the platform users to have a simplified representation of the environment to made and test changes and configurations more quickly and easily. Similarly, both for visualization and computation purposes, in case of multi-story buildings, it is beneficial to separate floors and create some logical links in the connectivity points such as stairs or elevators, easily controllable utilizing the related tools in Unity 3D.

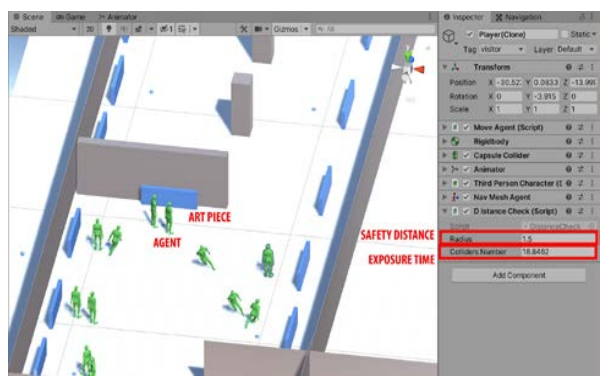

Figure 6: The simulation platform within the Unity 3D environment showing agents, art pieces and some of COVID-19 risk assessment parameters.

\section{CONCLUSIONS AND FUTURE DEVELOPMENTS}

The objective of the research presented in this paper is to develop a simulation approach, based on agent-based modeling, to support building use re-thinking within the COVID-19 infection. Although potentially extendable to any building typology, the model and the related platform have been mainly conceived and tested within the cultural buildings and museums scope, offering them an open and easy platform to favor their quick restart after this long period of forced non-operativity. As a result, the designers and owners receive a double-faces contribution to their rethink of the building and its use: on a qualitative level, they can visualize the transformed use phenomena, while on a quantitative level the risk index allows using metrics to compare different solutions. Relying on this simulation model, it is possible to virtually test different use hypothesis, investigating different options in terms of space layouts, users' behaviors, or both.

Although still under development, the presented approach is showing great efficacy and potentials in providing a quick way to assess the impact of use and design decisions in the context of cultural buildings and museums, allowing for a better comprehension of the adherence of the intended use scenarios to the COVID-19 infection risk reduction principles and norms. At the same time, some areas of further developments are emerging: a first is related to the possibility of integrating this kind of simulation with indoor air quality dynamic simulation, not only verifying the adherence to spatial norms but also the actual virus spreading phenomena with a better degree of accuracy; a second perspective is instead related to the adaptation of the same approach to other building typologies such as 
airports, schools and public buildings, where its adoption could provide benefits in the complex activity of re-thinking spaces and their use after the COVID-19 experience.

\section{REFERENCES}

Axelrod R. (1997). Advancing the Art of Simulation in the Social Sciences. In: Conte R., Hegselmann R., Terna P. (Eds) Simulating Social Phenomena. Lecture Notes in Economics and Mathematical Systems, vol 456. Springer, Berlin, Heidelberg.

Cheliotis K. (2020). An agent-based model of public space use. Computers, Environment and Urban Systems, Volume 81, 101476, ISSN 0198-9715.

Batty M. (2001). Agent-based pedestrian modeling. Environment and Planning B: Planning and Design, 28, 3, 321-326.

Bonabeau E. (2002). Agent-Based Modeling: Methods and techniques for simulating human systems. Proceedings of the National Academy of Sciences of the United States of America. 99 Suppl 3. 7280-7. 10.1073/pnas.082080899.

Carcellar B. G. (2017). Perception modelling of visitors in Vargas museum using agent-based simulation and visibility analysis. Int. Arch. Photogramm. Remote Sens. Spatial Inf. Sci., XLIIXLII-4/W5, 45-51.

Chu M. L., Parigi P., Latombe J. and Law, K. (2014) SAFEgress: A Flexible Platform to Study the Effect of Human and Social Behaviors on Egress Performance. Proc. SimAUD, 3542.4/W5, 45-51.

D'Orazio M., Bernardini G., Quagliarini E. (2020). How to restart? An agent-based simulation model towards the definition of strategies for COVID-19" second phase" in public buildings. ArXiv Preprint ArXiv:2004.12927.

Goscé L., Barton, D., Johansson, A. (2015). Analytical Modelling of the Spread of Disease in Confined and Crowded Spaces. Sci Rep 4, 4856.

Honey-Roses J., Anguelovsk, I., Bohiga, J., Chire, V.,Daher, C., Konijnendijk, C., ... Nieuwenhuijsen, M. (2020). The Impact of COVID-19 on Public Space: A Review of the Emerging Questions. https://doi.org/10.31219/osf.io/rf7xa.

ICOM - International Council of Museums, 2020, Museums, museum professionals and COVID-19: Survey results. Retrieved from https://icom.museum/en/news/museumsmuseum-professionals-and-COVID-19-survey-results/.

COVID-19 - Che cos'è il nuovo coronavirus (2020). Retrieved from http://www.salute.gov.it/portale/nuovocoronavirus/dettaglioFa qNuovoCoronavirus.jsp?lingua=italiano\&id=228.

McKinsey Report Reimagining the office and work life after COVID-19. Retrieved from https://www.mckinsey.com/businessfunctions/organization/our-insights/reimagining-the-officeand-work-life-after-COVID-19.
Perez L, Dragicevic S. (2009). An agent-based approach for modeling dynamics of contagious disease spread. Int J Health Geogr. 2009;8:50. doi:10.1186/1476-072X-8-50.

Pluchino A., Garofalo C., Inturri G., Rapisarda A., Ignaccolo M. (2014). Agent-Based Simulation of Pedestrian Behaviour in Closed Spaces: A Museum Case Study. Journal of Artificial Societies and Social Simulation. doi: 17. 10.18564/jasss.2336.

Ronchi E., Lovreglio R,( 2020). EXPOSED: An occupant exposure model for confined spaces to retrofit crowd models during a pandemic. arXiv preprint arXiv:2005.04007. doi:2005.04007.

Simeone D. and Kalay Y. E. (2012). An Event-Based Model to simulate human behaviour in built environments. In Digital Physicality: Proceedings of the 30th eCAADe Conference, Prague, Czech Republic, pp. 525-532.

Simeone D., Cursi S., Coraglia U.M. (2017): Modelling buildings and their use as systems of agents. In ShoCK! - Sharing Computational Knowledge! - Proceedings of the 35th eCAADe Conference - Volume 1, Sapienza University of Rome, Rome, Italy, 20-22 September 2017, pp. 85-92.

Simeone D., Cursi S., Acierno M. (2019): BIM semanticenrichment for built heritage representation. Automation in Construction, Volume 97, 122-137.

Tabak V. (2008). User Simulation of Space Utilisation - System for Office Building Usage Simulation, PhD Thesis, Eindhoven University of Technology, Eindhoven, Netherlands.

UNESCO, 2020, Museums around the world in the face of COVID-19, Paris. Retrieved from https://unesdoc.unesco.org/ark:/48223/pf0000373530.

World Health Organization, 2020, World Health Organization. (2020). Considerations for school-related public health measures in the context of COVID-19: annex to considerations in adjusting public health and social measures in the context of COVID-19, 10 May 2020. World Health Organization. Retrieved from https://apps.who.int/iris/handle/10665/332052. License: CC BY-NC-SA 3.0 IGO

Wurzer G. (2010). Schematic Systems - Constraining Functions Through Processes (and Vice Versa). International Journal of Architectural Computing 08 (02), pp. 197-214.

Zimmermann G. (2010). Agent-Based Modeling and Simulation of Individual Building Occupants. In Proceedings of SimBuild 2010 Conference: IBPSA-USA, 269-276.

Williams, J. H. (2008). Employee engagement: Improving anticipation in safety. Professional Safety, 53(12), 40-45.

Keller, T. E., Cusick, G. R., \& Courtney, M. E. (2007). Approaching the transition to adulthood: Distinctive profiles of adolescents aging out of the child welfare system. Social Services Review, 81, 453- 484 . 